\title{
Accuracy of molecular diagnostics in pemphigus and bullous pemphigoid: comparison of commercial and modified mosaic indirect immunofluorescence tests as well as enzyme-linked immunosorbent assays
}

\author{
Justyna Gornowicz-Porowska1, Agnieszka Seraszek-Jaros², Monika Bowszyc-Dmochowska³, Elżbieta Kaczmarek², \\ Paweł Pietkiewicz ${ }^{1}$, Paweł Bartkiewicz ${ }^{1}$, Marian Dmochowski ${ }^{1}$

\begin{abstract}
${ }^{1}$ Autoimmune Blistering Dermatoses Section, Department of Dermatology, Poznan University of Medical Sciences, Poznan, Poland 2Department of Bioinformatics and Computational Biology, Poznan University of Medical Sciences, Poznan, Poland

${ }^{3}$ Cutaneous Histopathology and Immunopathology Section, Department of Dermatology, Poznan University of Medical Sciences,
\end{abstract} \\ Poznan, Poland
}

Adv Dermatol Allergol 2017; XXXIV (1): 21-27

DOI: https://doi.org/10.5114/ada.2017.65617

\begin{abstract}
Introduction: Pemphigus and bullous pemphigoid (BP) are identified by autoantibodies (abs) against desmoglein 1, 3 (DSG1/3) and BP180/BP230, respectively. A novel mosaic to indirect immunofluorescence (IIF) using purified BP180 recombinant proteins spotted on slide and transfected cells expressing BP230, DSG1, DSG3 is available. The commercial (IgG detection) and modified (IgG4 detection) mosaic for indirect immunofluorescence (IIFC - IIF commercial, IIFm - IIF modified) and IgG ELISAs were evaluated in pemphigus and bullous pemphigoid (BP) molecular diagnostics. Aim: To compare diagnostic accuracy of commercial (IgG detection) and modified (IgG4 detection) mosaic IIF assay and to examine the diagnostic value of ELISAs in relation to mosaic IIF in routine laboratory diagnostics of pemphigus and BP.

Material and methods: Sera from $37 \mathrm{BP}$ and 19 pemphigus patients were studied. Associations between tests were assessed using Fisher's exact test.

Results: There are associations between the positive/negative samples detected by IIFc with desmoglein1 (DSG1)/ desmoglein3 (DSG3)/BP230 transfected cells and ELISAs and no association between anti-BP180 IgG detection by IIFC and ELISA. IIFm with DSG1 and DSG3 showed both $100 \%$ sensitivity and $100 \%$ and $78 \%$ specificity, respectively, and $100 \%$ and $83 \%$ positive predictive value in relation to IIFc. IIFm with BP230 had $87 \%$ specificity, $55 \%$ sensitivity, whereas IIFm with BP180 had a $100 \%$ sensitivity and $13 \%$ specificity in relation to IIFc.

Conclusions: The IIFc with DSG1/DSG3/BP230 transfected cells, excluding BP180 spots, is an alternative method to ELISA in pemphigus/BP diagnostics. IgG4 antibodies, both pathogenically and diagnostically important, are inconsistently detectable with IIFm.
\end{abstract}

Key words: pemphigus, pemphigoid, bullous, immunologic tests.

\section{Introduction}

Autoimmune blistering dermatoses (ABD) are immunologically characterized by the pathological autoimmune responses directed against cell-cell or cell-matrix adhesion molecules [1]. More common ABD entities, pemphigus and bullous pemphigoid (BP), are identified by circulating and tissue-bound autoantibodies (abs) against the desmosomal cadherins (mainly desmoglein 1 and 3 -DSG1/3) and dermal-epidermal junction components (BP180 and BP230), respectively. Autoimmunity in these dermatoses is associated with stimulation of Th1/Th2 cells responsible for pathogenic abs production by B cells and these patients may contain Th1-regulated IgG1 and Th2-regulated IgG4 abs directed against DSG1/DSG3 or BP180/BP230, respectively [2]. Detected abs usually belong to the IgG class, however the significance of IgG1 and IgG4 subclasses seems to be still equivocal.

Address for correspondence: Justyna Gornowicz-Porowska PhD, MSc, Autoimmune Blistering Dermatoses Section, Department of Dermatology, Poznan University of Medical Sciences, 49 Przybyszewskiego St, 60-355 Poznan, Poland, phone: +48 603 222 280, e-mail: justynagornowicz1@poczta.onet.pl Received: 16.09.2015, accepted: 29.11.2015. 
Recently, the complex and heterogeneous pathogenesis of $A B D$ has been revealed. Clinical heterogeneity determines that $A B D$ can mimic a variety of clinical conditions with unusual variants of disease, including non-bullous clinical manifestations [3]. In light of this, isotype/class switching, autoantibody shift, enhanced immunogenicity of antigens by specific triggering factors (including drugs) [4] through the exposition of sequestered antigens to the immune system as well as conformational changes may underline the molecular heterogeneity of ABD. Thus, clinical and molecular heterogeneity of $A B D$ poses a surprisingly great diagnostic challenge to the dermatologists making their diagnostic work a complex multistep process.

Nowadays, the diagnosis of ABD is generally based on a combination of various criteria: clinical features, histology and immunological tests, notably direct and indirect immunofluorescence (DIF and IIF, respectively) and enzyme linked immunosorbent assay (ELISA) [5]. The precise identification of the target antigen is crucial for patient management. Several assays for the serological detection of anti-DSG1/DSG3/BP180/BP230 abs have been developed, however no generally accepted gold standard assay exists, and thus a working set for as definitive diagnosis as possible is urgently needed.

Recently, novel techniques for serological ABD diagnosis, such as biochip mosaic-based and bioplex-based techniques, have been discussed [6-9]. A novel IIF microscopy approach using purified BP180 recombinant proteins spotted on slide and transfected cells expressing BP230, DSG1, DSG3 is available.

Here, we describe a modified BIOCHIP mosaic-based indirect immunofluorescence (modified indirect immunofluorescence - IIFm) technique for the determination of anti-DSG1, anti-DSG3, anti-BP180 and anti-BP230 IgG4 abs based on recombinant antigenic substrates and transfected cells in relation to commercial BIOCHIP mosaic-based IIF (commercial indirect immunofluorescence - IIFc) for the determination IgG abs. In this study we investigated the sensitivity, specificity as well as positive and negative predictive values of this innovative immunoassay in order to evaluate its diagnostic use for the laboratory diagnosis of ABD compared with ELISAs.

\section{Aim}

The aim of this study was to compare the diagnostic performance of original and modified multiparametric IIF assay for ABD serology. We attempted to modify original mosaic-based IIF for IgG to pemphigus and BP antigens (DSG1/DSG3/BP180/BP230) in order to enable assessment of IgG4 abs to those antigens.

The second aim was to examine the diagnostic value of commercial ELISAs in relation to mosaic-based IIF with transfected cells in routine laboratory diagnostics of ABD in a setting in Poland.

\section{Material and methods}

This study was approved by the local Ethical Committee of the Poznan University of Medical Sciences in Poland and informed written consent was obtained from each individual.

\section{Patients and serum samples}

Altogether, 56 patients with ABD before initiation of treatments were investigated. The examined group involved 19 pemphigus patients (pemphigus vulgaris and pemphigus foliaceus) and 37 BP patients. Patients in the examined groups met following criteria: (i) clinical features - flaccid blisters and erosions on the skin and mucous membranes in pemphigus; tense cutaneous blisters with no or transient involvement of mucosal surfaces and sparing head and neck in BP, (ii) typical immunoglobulin deposits detected with DIF of perilesional skin, (iii) molecular characterization of antigens. The diagnosis of BP was made in patients having IgG/lgG4 and/or IgG1 nonU-pattern deposits along the dermal-epidermal junction in DIF, diagnosis was corroborated with positive BP180 and/or BP230 IgG ELISA in serum samples. Other subepidermal autoimmune blistering dermatoses could be excluded based on these data. The diagnosis of pemphigus was made in patients having lgG/lgG4/lgG1 fish-net like or dewdrops on spider web patterns in DIF, diagnosis was corroborated with DSG1/DSG3 IgG ELISA in serum samples. The diagnosis of ABD was supported by histology.

Patients were recruited during the period of October 2013 through February 2015 at the Autoimmune Blistering Dermatoses Section, Department of Dermatology, Poznan University of Medical Sciences, Poland. The serum used in the serological tests was taken at the time of hospital admission/ambulatory care. Five $\mathrm{ml}$ of blood serum were obtained from each participant. The samples were centrifuged for $10 \mathrm{~min}$ at $3500 \mathrm{rpm}$. Thereafter, they were stored at $-20^{\circ} \mathrm{C}$ until performing ELISA and BIOCHIP mosaic IIF.

\section{ELISA}

The specific circulating serum abs were detected with commercially available ELISAs. ELISAs were performed using the Euroimmun (Luebeck, Germany) ELISA kits, utilizing recombinant protein DSG1, DSG3, BP180, BP230, with the manufacturer's cut-off value of $20 \mathrm{RU} / \mathrm{ml}$. AntiBP180-NC16A-4X ELISA includes four copies of domain NC16A fused to a polyhistidine tag to enhance protein expression. Anti-BP230-CF ELISA contains an amplified fragment of C-terminal globular domain. Anti-DSG1 IgG and anti-DSG3 IgG was measured with an ELISA assay utilizing recombinant proteins DSG1 and DSG3, consisting of the extracellular domain of DSG1 and DSG3, respectively ( 5 subdomains). All measurements were made in the ELISA plate reader (Asys Expert 96) equipped with 

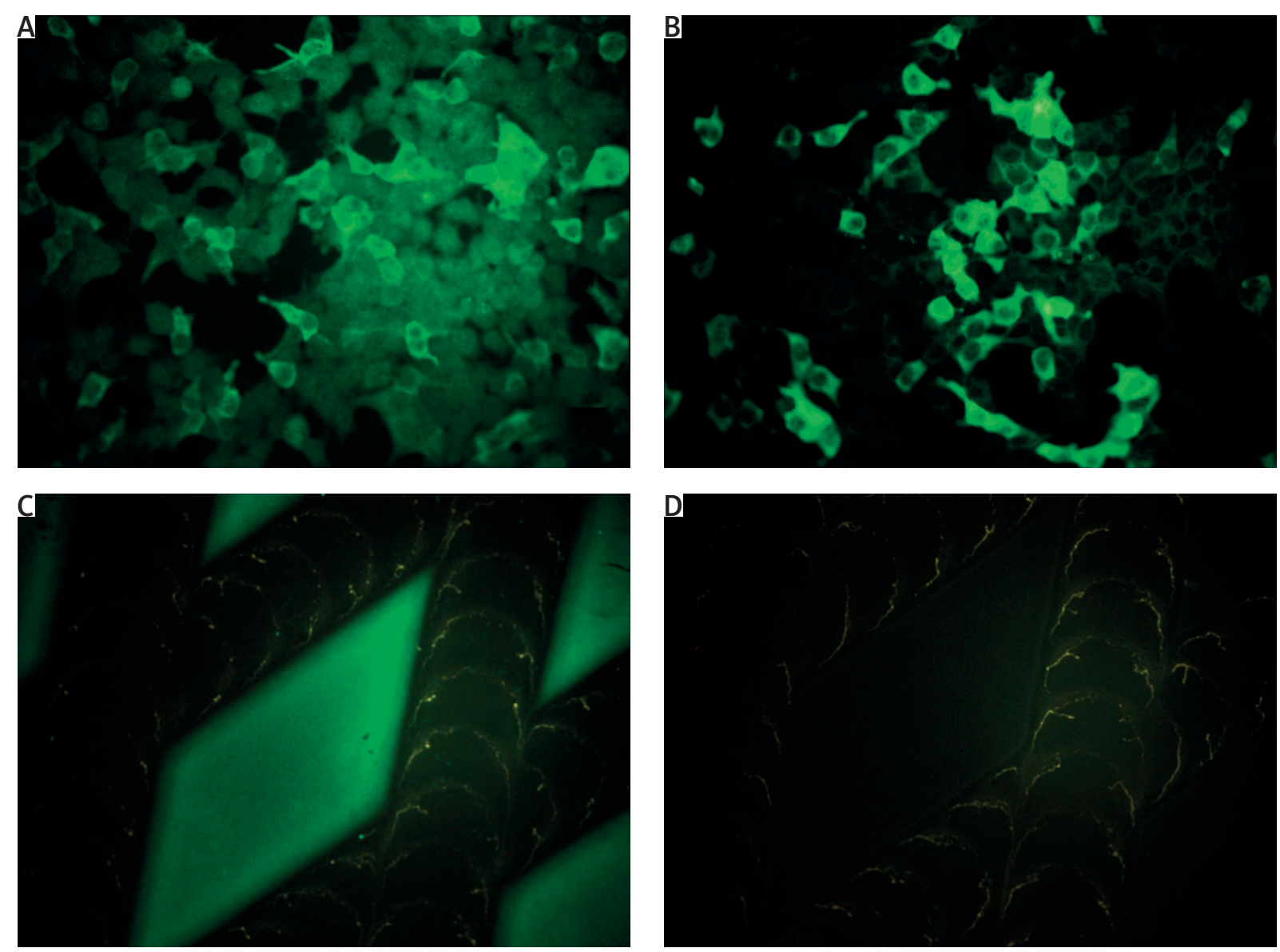

Figure 1. Indirect immunofluorescence mosaic for autoimmune blistering dermatoses in a 78-year-old woman with bullous pemphigoid (DIF of perilesional skin revealed linear IgG1, IgG4, C3 deposits along the dermal-epidermal junction; ELISA serum anti-BP180-NC16A-4X IgG $172.809 \mathrm{RU} / \mathrm{ml}$, anti-BP230-C IgG above $200 \mathrm{RU} / \mathrm{ml}$, cut-off $20 \mathrm{RU} / \mathrm{ml}$ ): positive reaction with IgG (A), IgG4 (B) autoantibodies against BP230-C expressed in transfected HEK293 cells and positive reaction with IgG (C), but negative reaction with IgG4 (D), autoantibodies against the tetrameric BP180 NC16A spots (antigen dots)

Microwin 2000 software by a single operator following the manufacturer's instructions.

\section{BIOCHIP mosaic IIF}

BIOCHIP-mosaic based IIF (EUROPLUS Dermatology Mosaic, Euroimmun, Luebeck, Germany) consists of an array of six different diagnostic substrates including monkey esophagus, primate salt-split skin, dots of tetrameric BP180-NC16A as well as DSG1 and DSG3 extracellular and transmembrane domains (E + TM) and BP230 C-terminal domain expressed in HEK293 (human embryonic kidney) cells. The reaction was performed following the manufacturer's instructions. The original BIOCHIP with the assessment of anti-DSG1/DSG3/BP180/BP230 lgG was modified to enable assessment of IgG4 abs to those antigens (Figures 1 A-D, Figures 2 A-D). The IIFm was provided by replacing the standard fluorescein-conjugated anti-human IgG (Euroimmun, Germany) by a mouse monoclonal anti-human fluorescein-conjugated IgG4 secondary an- tibody (1: 100 dilution; Sigma, USA). The procedure of IIF consisted of two consecutive incubation steps. Firstly, diluted $(1: 10)$ serum samples were incubated for $30 \mathrm{~min}$ at room temperature (RT). In the second 30 min at RT the attached autoantibodies are stained with fluoresceinlabeled anti-human IgG, IgG4 antibodies.

All slides were evaluated simultaneously by two observers using a microscope (BX40, Olympus, Japan) with a fluorescent adapter.

\section{Statistical analysis}

The accuracy of modified mosaic IIF and ELISA was evaluated by calculating diagnostic sensitivity, diagnostic specificity as well as positive and negative predictive values in relation to the original/commercial mosaic IIF using the MedCalc Software 2015 (Ostend, Belgium; www. medcalc.org). Associations in results between tests were assessed using Fisher's exact test. A $p<0.05$ was arbitrarily considered statistically significant. 

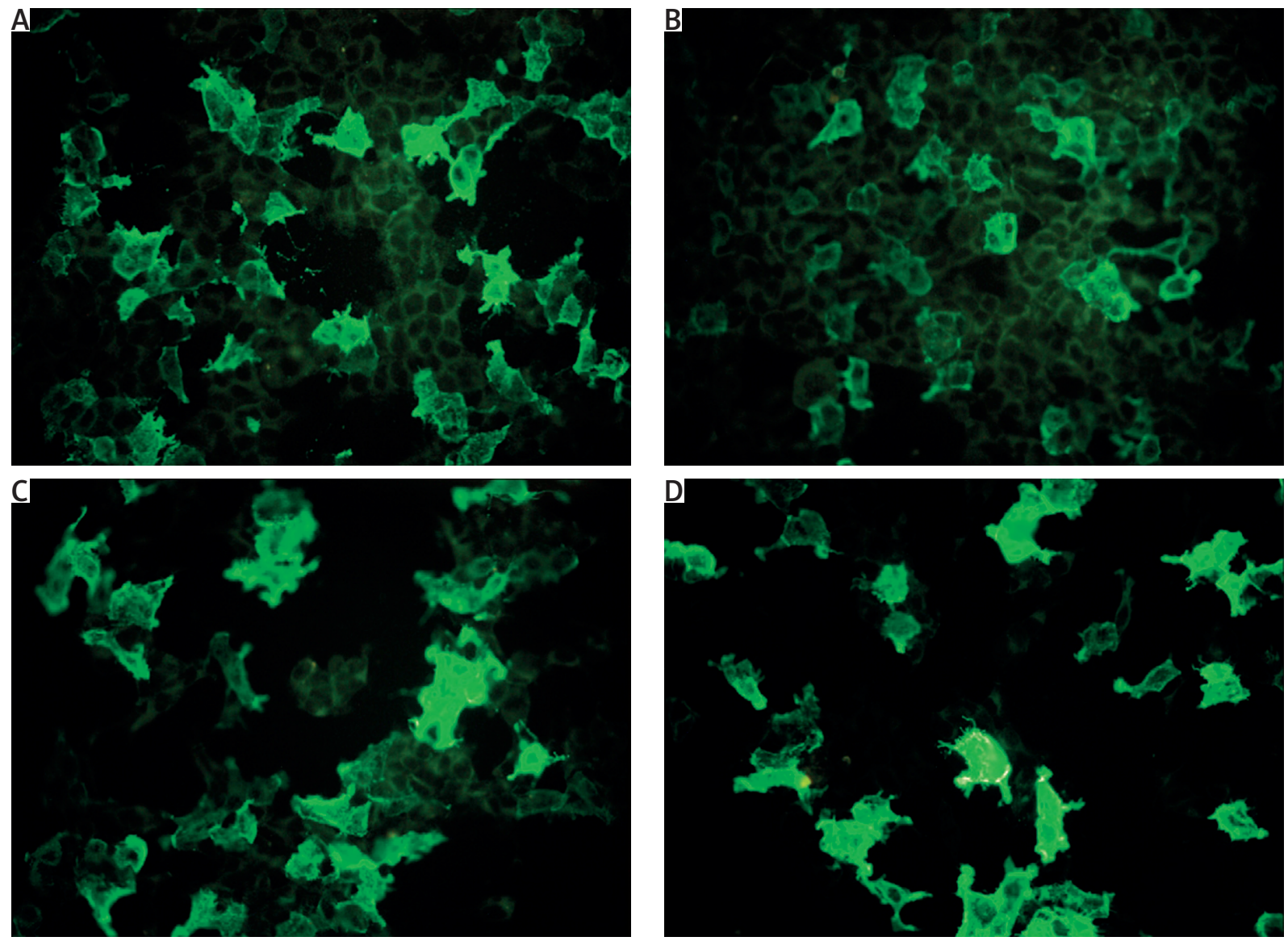

Figure 2. Indirect immunofluorescence mosaic for autoimmune blistering dermatoses in a 71-year-old woman with mucocutaneous pemphigus vulgaris (DIF of perilesional skin revealed pemphigus-type IgG1, IgG4, C3 deposits in the epidermis; ELISA serum anti-DSG1 IgG 91. $593 \mathrm{RU} / \mathrm{ml}$, anti-DSG3 lgG above $200 \mathrm{RU} / \mathrm{ml}$, cut-off $20 \mathrm{RU} / \mathrm{ml}$ ): positive reaction with lgG (A), IgG4 (B) autoantibodies against extracellular and transmembrane domains of DSG1 expressed in transfected HEK293 cells and positive reaction with IgG (C), IgG4 (D) autoantibodies to extracellular and transmembrane domains of DSG3 expressed in transfected HEK293

\section{Results}

Comparison of diagnostic accuracy between modified (IgG4) mosaic IIF and original (IgG) mosaic IIF

The diagnostic sensitivity and specificity as well as positive and negative predictive values of modified (IgG4) in comparison with standard (IgG) IIF are shown in Table 1.

Comparison of diagnostic accuracy between original (IgG) mosaic IIF and ELISA

The diagnostic sensitivity and specificity as well as positive and negative predictive values of original mosaic (IgG) IIF in relation to ELISA are shown in Table 2.

Associations between modified (IgG4), original (IgG) mosaic IIF and ELISA

There is an association between the values of positive/negative samples detected by original/commercial
IIF with DSG1/DSG3/BP230 transfected cells and ELISAs $(p<0.05)$. There is no association between anti-BP180 IgG detection in BP patients by IIF and ELISA ( $p=0.1081)$.

There is an association between the values of positive/negative samples detected by modified (IgG4) mosaic IIF with DSG1/DSG3/BP230 transfected cells and original/commercial (IgG) mosaic IIF ( $p<0.05)$ (Figures 1 A, B, Figures 2 A-D).

There is no association between anti-BP180 lgG4 detected by modified IIF and anti-BP180 IgG detected by original/ commercial mosaic IIF ( $p=0.5894$ ) (Figures 1C, D).

\section{Discussion}

To date, no previous study has stringently compared the two mosaic IIF assays (commercially available and innovative modification to characterize the IgG4 reactivity in sera considering that the production of abs in pemphigus and BP is polyclonal) in terms of their use in the diagnosis of pemphigus and BP in a defined population. 
Table 1. Calculation of the diagnostic sensitivity, specificity and predictive values of multiparametric IIF (modified versus original)

\begin{tabular}{|c|c|c|c|c|c|c|c|c|c|}
\hline \multicolumn{10}{|c|}{ Diagnostic accuracy of modified mosaic IIF in relation to original mosaic IIF } \\
\hline \multirow{2}{*}{$\begin{array}{l}\text { Modified (IgG4) } \\
\text { versus original } \\
\text { (IgG) IIF }\end{array}$} & \multirow[t]{2}{*}{$\begin{array}{l}\text { Subjects } \\
\qquad(n)\end{array}$} & \multicolumn{2}{|c|}{$\begin{array}{c}\text { Number of } \\
\text { positive results }\end{array}$} & \multicolumn{2}{|c|}{$\begin{array}{c}\text { Number of } \\
\text { negative results }\end{array}$} & \multirow[t]{2}{*}{$\begin{array}{c}\text { Sensitivity } \\
(\%)\end{array}$} & \multirow[t]{2}{*}{$\begin{array}{l}\text { Specificity } \\
(\%)\end{array}$} & \multirow{2}{*}{$\begin{array}{c}\text { Positive } \\
\text { predictive value } \\
\text { (PPV) (\%) }\end{array}$} & \multirow{2}{*}{$\begin{array}{l}\text { Negative } \\
\text { predictive value } \\
\text { (NPV) (\%) }\end{array}$} \\
\hline & & $\lg G$ & $\operatorname{lgG4}$ & $\operatorname{lgg}$ & $\operatorname{lgG} 4$ & & & & \\
\hline DSG1 & 19 & 13 & 13 & 6 & 6 & 100 & 100 & 100 & 100 \\
\hline DSG3 & 19 & 12 & 10 & 7 & 9 & 100 & 78 & 83 & 100 \\
\hline BP180 & 37 & 33 & 7 & 4 & 30 & 100 & 13 & 21 & 100 \\
\hline BP230 & 37 & 9 & 6 & 28 & 31 & 55 & 78 & 62 & 84 \\
\hline
\end{tabular}

IIF - indirect immunofluorescence, DSG1 - desmoglein 1, DSG3 - desmoglein 3, BP180 - bullous pemphigoid antigen 180, BP230 - bullous pemphigoid antigen $230, n$-number of patients.

Table 2. Calculation of the diagnostic sensitivity, specificity and predictive values of original multiparametric IIF in relation to ELISA

\begin{tabular}{|c|c|c|c|c|c|c|c|}
\hline \multicolumn{8}{|c|}{ Diagnostic accuracy of original mosaic IIF in relation to ELISA } \\
\hline \multirow{2}{*}{$\begin{array}{l}\text { Original mosaic IIF (IgG) } \\
\text { versus ELISA (IgG) }\end{array}$} & \multirow{2}{*}{$\begin{array}{l}\text { Subjects } \\
(n)\end{array}$} & \multicolumn{2}{|c|}{ Results of ELISA } & \multirow{2}{*}{$\begin{array}{c}\text { Sensitivity } \\
\text { (\%) }\end{array}$} & \multirow{2}{*}{$\begin{array}{l}\text { Specificity } \\
\text { (\%) }\end{array}$} & \multirow{2}{*}{$\begin{array}{l}\text { Positive } \\
\text { predictive value } \\
\text { (PPV) (\%) }\end{array}$} & \multirow{2}{*}{$\begin{array}{l}\text { Negative } \\
\text { predictive value } \\
(\mathrm{NPV})(\%)\end{array}$} \\
\hline & & $\begin{array}{c}\text { Number of } \\
\text { positive results }\end{array}$ & $\begin{array}{c}\text { Number of } \\
\text { negative results }\end{array}$ & & & & \\
\hline DSG1 & 19 & 12 & 7 & 100 & 85 & 92 & 100 \\
\hline DSG3 & 19 & 13 & 6 & 100 & 85 & 92 & 100 \\
\hline BP180 & 37 & 36 & 1 & 91 & 100 & 100 & 25 \\
\hline BP230 & 33 & 9 & 24 & 55 & 87 & 62 & 84 \\
\hline
\end{tabular}

Moreover, in extension of previous findings, our study investigated mosaic IIF with DSG1/DSG3/BP230 transfected cells and BP180 NC16A spots as an alternative way to ELISAs in management of pemphigus and BP patients.

Our results indicated that transfected cells may be useful, with the diagnostic accuracy similar to ELISAs, in laboratory diagnostics of pemphigus and BP supporting previous investigations [6-9]. A novel finding of our study was the infrequent detection of BP180-reactive IgG4 on BP180 NC16A spots with IIF. However, it should be noted that data obtained by various authors depended on the applied antigen recombinants, the selected patients and the methodology used.

Our previous observations with the use of DIF and IIF confirm that circulating abs in pemphigus and BP belong predominantly to the IgG4 isotype $[2,10]$. However, the molecular specificity of detected abs was not documented there. Thus, here the molecular characterization of antigens in relation to IgG and IgG4 abs was also investigated.

The diagnostic accuracy of the transfected cells with DSG1 and DSG3 detecting IgG4 compared favourably with that of the standard transfected cells detecting IgG abs. Modified mosaic IIF with DSG1 and DSG3 showed a high sensitivity (100\%), specificity $(100 \%$ and $78 \%$, respectively) and positive predictive value (100\% and $83 \%$, respectively) suggesting that the use of the modified mosaic IIF may improve the diagnosis of pemphigus in health facilities. Our data are consistent with other researchers showing the preferential detection of anti-DSG reactive IgG4 in active pemphigus vulgaris (PV) [11]. Thus, probably the use of transfected cells with DSGs might have facilitated their detection. Previous findings indicated that IgG4 subclass is probably implicated in the pathogenesis of pemphigus foliaceus (PF) and passive transfer of patients IgG4 abs has been shown to produce disease in neonatal mice $[12,13]$. Interestingly, some reports demonstrated that the anti-DSG1 abs in PV are pathogenic and have IgG4 subclass specificity [14].

In case of BP patients, our results may confirm the thesis that Th2-regulated abs preferentially bind to BP230 in BP patients. The diagnostic accuracy of the transfected cells with BP230 detecting IgG4 had a good specificity (87\%) but a poor sensitivity (55\%) compared with that of the standard transfected cells detecting IgG abs. Interestingly, BP180 NC16A spots revealed a good sensitivity for IgG and IgG4 detection but at the expense of specificity. BP180 NC16A spots showed a high sensitivity (100\%) but very low specificity (13\%) and positive predictive value (21\%). These findings confirm the previous study indicating Th-2 response as preferentially directed against BP230 [15]. Results obtained by Ghohestani et al. [15] using different antigenic sources showed IgE abs as directed exclusively against BP230, and not 
against BP180 or any other epidermal or dermal antigens. It is known that IgG4 and IgE production is regulated concordantly and requires the stimulation of Th2 cells [16]. Probably, IgG and IgG4 from BP sera were directed against the same epitope on the C-terminal end of the protein suggesting immunoglobulin class switching from IgG to IgG4. Here, the detection of anti-BP230 IgG4 abs strengthens the link between autoimmune pemphigoid and immune response involving Th2 cells.

Remarkably, in contrast to BP230, BP180 is not the target of IgG4 in our study. The lack of IgG4 reactivity to BP180 is not due to loss of antigen immunoreactivity, as it was clearly labeled by IgG abs from the same serum samples. Moreover, in some sera containing IgG against both BP230 and BP180, only IgG4 against BP230 was detected. Thus, the possibility of technical differences (various methods of BP230 and BP180 recombination) in the detection of IgG4 abs should be taken into consideration.

Our observations are contradictory with Döpp et al. [17] who analyzed the IgG subclass distribution to fulllength BP180 NC16A and recombinant fragments of this domain with the use of ELISA and IB and showed that IgG4 and IgE are the major isotypes of immunoglobulin targeting BP180 NC16A. Our finding that anti-BP180 IgG4 is a nonpredominant subclass is also in contrast with Modre et al. [18] who observed this in remission, but not at the early stage of the disease. Revealed discrepancies may result from different research methods used in the studies exhibiting variable sensitivity and specificity or from detection of different antigenic regions of the examined protein. It is known that the structure of an antibody is related to its function. Thus, the protein purification and immobilization may change in protein conformation (the effect on tertiary structure of the protein) as well as the antigen alternations may have an impact on binding specificity and/or biological activity. The method used here (BP180 NC16A spots) significantly decreased the protein affinity in comparison to other diagnostic techniques - probably by low antibody avidity or loss of the antigenic structure during "spots" creation/development.

\section{Conclusions}

The most appropriate choice of a diagnostic assay for molecular characterization of autoimmunity may depend on the clinical setting. Interestingly, in this respect, it has been suggested using a BP180 humanized mouse model [19] that IgG4 abs, which should be regarded as a diagnostically useful biomarker of an active Th2-mediated autoimmunity, to BP180 NC16A may be protective, which may simply reflect the fact that the stereotypically elderly BP-affected individual is still able to cope with the disease at its active stage.

Findings obtained to date seem to indicate divergent roles for lgG4, characterized as an odd [20] subclass, abs in BP and pemphigus development, what may have an impact on disease severity (idiopathic BP as such is a less serious illness than idiopathic pemphigus).

The continuation of efforts to modify the original IIF test to enable the evaluation of IgG4 abs to ABD antigens seems justified. It seems that IIF with DSG1/DSG3/ BP230 transfected cells is an alternative method to ELISA in management of pemphigus/BP patients. However, for anti-BP180 IgG detection in BP patients the ELISA remains a definitive serological test. IgG4, both pathogenically and diagnostically important, are inconsistently detectable with IIFm. IIFm may help diagnosing serologically challenging cases.

\section{Acknowledgments}

A part of this study was accepted for presentation at the $45^{\text {th }}$ Annual ESDR Meeting (9-12 September 2015, Rotterdam, The Netherlands) Gornowicz-Porowska J, Bowszyc-Dmochowska M, Pietkiewicz P, Seraszek-Jaros A, Kaczmarek E, Bartkiewicz P., Dmochowski M. Comparative diagnostic performance of original as well as modified multiparametric immunofluorescence assay and ELISAs for the serological detection of pemphigus and bullous pemphigoid activity (no. 089).

This study was partly funded from grant of the Polish Ministry of Science and Higher Education no. 0127/ IPI/2015/73.

BIOCHIP mosaic was obtained from EUROIMMUN (Germany) within a framework of a multicenter project: "A European prospective study on serum antibodies against target antigens of bullous autoimmune diseases and genetic susceptibility".

\section{Conflict of interest}

The authors declare no conflict of interest.

\section{References}

1. Gornowicz-Porowska J, Bowszyc-Dmochowska M, Dmochowski M. Autoimmunity-driven enzymatic remodeling of the dermal-epidermal junction in bullous pemphigoid and dermatitis herpetiformis. Autoimmunity 2012; 45: 71-80.

2. Gornowicz-Porowska J, Pietkiewicz P, Bowszyc-Dmochowska M, Dmochowski M. Immunoglobulin G4 is prevailing over immunoglobulin G1 in autoimmunity of pemphigus and bullous pemphigoid: analysis of tissue-bound antibodies in active diseases. Centr Eur J Immunol 2013; 38: 80-91.

3. Męcińska-Jundziłt K, Hashimoto T, Kowalewski C, et al. Discrepancies among clinical, histological and immunological findings in IgA pemphigus: a case report and literature survey. Adv Dermatol Allergol 2016; 33: 480-4.

4. Pietkiewicz P, Gornowicz-Porowska J, Bowszyc-Dmochowska M, Dmochowski M. A retrospective study of antihypertensives in pemphigus: a still unchartered odyssey particularly between thiols, amides and phenols. Arch Med Sci 2015; 11: 1021-7.

5. Schmidt E, Zillikens D. Modern diagnosis of autoimmune blistering skin diseases. Autoimmun Rev 2010; 10: 84-9. 
6. van Beek N, Rentzsch K, Probst C, et al. Serological diagnosis of autoimmune bullous skin diseases: prospective comparison of the BIOCHIP mosaic-based indirect immunofluorescence technique with the conventional multi-step single test strategy. Orphanet J Rare Dis 2012; 7: 49.

7. Damoiseaux J, van Rijsingen M, Warnemünde N, et al. Autoantibody detection in bullous pemphigoid: clinical evaluation of the EUROPLUS ${ }^{\text {TM }}$ Dermatology Mosaic. J Immunol Methods 2012; 382: 76-80.

8. Russo I, Saponeri A, Peserico A, Alaibac M. The use of biochip immunofluorescence microscopy for the diagnosis of pemphigus vulgaris. Acta Histochem 2014; 116: 713-6.

9. Zarian H, Saponeri A, Michelotto A, et al. Biochip technology for the serological diagnosis of bullous pemphigoid. ISRN Dermatol 2012; 2012: 237802.

10. Pietkiewicz P, Gornowicz-Porowska J, Dmochowski M, Bowszyc-Dmochowska M. Serum IgG4 antibody examination in patients with IgG-mediated autoimmune subepidermal blistering dermatoses increases autoimmunity detection. XXX Convention of Polish Society of Dermatology, 19-22 September 2012, Cracow, Poland. Przegl Dermatol 2012; 99: 520.

11. Spaeth S, Riechers R, Borradori L, et al. IgG, IgA and IgE autoantibodies against the ectodomain of desmoglein 3 in active pemphigus vulgaris. Br J Dermatol 2001; 144: 1183-8.

12. Allen EM, Giudice GJ, Diaz LA. Subclass reactivity of pemphigus foliaceus autoantibodies with recombinant human desmoglein. J Invest Dermatol 1993; 100: 685-91.

13. Rock B, Martins CR, Theofilopoulos AN, et al. The pathogenic effect of IgG4 autoantibodies in endemic pemphigus foliaceus (fogo selvagem). N Engl J Med 1989; 320: 1463-9.

14. Ding X, Diaz LA, Fairley JA, et al. The anti-desmoglein 1 autoantibodies in pemphigus vulgaris sera are pathogenic. J Invest Dermatol 1999; 112: 739-43.

15. Ghohestani RF, Cozzani E, Delaporte E, et al. IgE antibodies in sera from patients with bullous pemphigoid are autoantibodies preferentially directed against the 230-kDa epidermal antigen (BP230). J Clin Immunol 1998; 18: 202-9. Erratum in: J Clin Immunol 1998; 18: 441.

16. Bowszyc-Dmochowska M. Studies on pathogenesis of bullous pemphigoid. Ph.D. Doctoral thesis, Poznan University of Medical Sciences, Poznan, Poland, 2001.

17. Döpp R, Schmidt E, Chimanovitch I, et al. IgG4 and IgE are the major immunoglobulins targeting the NC16A domain of BP180 in bullous pemphigoid: serum levels of these immunoglobulins reflect disease activity. J Am Acad Dermatol 2000; 42: 577-83.

18. Modre B, Allen J, Wojnarowska F. Does class switching contribute to remission in bullous pemphigoid? Acta Derm Venereol 1999; 79: 127-31.

19. Zuo Y, Evangelista F, Guilabert A, et al. Anti-BP180 lgG4 autoantibodies are inhibitory in BP in BP180 humanized mouse model. 2015 SID Annual Meeting, 6-9 May 2015 Atlanta, USA. J Invest Dermatol 2015; 135 (Suppl. 1): S16.

20. Aalberse RC, Stapel SO, Schuurman J, Rispens T. Immunoglobulin G4: an odd antibody. Clin Exp Allergy 2009; 39: 469-77. 